\title{
Capturing Entertainment through Heart Rate Dynamics in the Playware Playground
}

\author{
Georgios N. Yannakakis, John Hallam and Henrik Hautop Lund \\ Maersk Institute for Production Technology \\ University of Southern Denmark \\ \{georgios, john, hhl\}@mip.sdu.dk
}

\begin{abstract}
This paper introduces a statistical approach for capturing entertainment in real-time through physiological signals within interactive playgrounds inspired by computer games. For this purpose children's heart rate (HR) signals and judgement on entertainment are obtained from experiments on the innovative Playware playground. A comprehensive statistical analysis shows that children's notion of entertainment correlates highly with their average HR during the game.
\end{abstract}

\section{Introduction}

Motivated by the lack of quantitative models of entertainment, an endeavor for capturing player satisfaction in real-time through physiological signals during gameplay is introduced in the work presented here. In this work, we define entertainment primarily as the level of satisfaction generated by the real-time playergame opponent interaction - by 'opponent' we define any controllable interactive feature on the game. Even though entertainment is a highly complicated mental state it is correlated with sympathetic arousal which can be captured through specific physiological signals such as heart rate and skin conductivity reported by researchers in the psychophysiological research field. Herein we investigate the impact of player satisfaction on heart rate (HR) signals and attempt to capture HR signal features that correlate with human notion of entertainment. HR signal data and children's judgement on entertainment are obtained through a gaming experiment devised on the Playware playground. The 'Playware' [4] intelligent interactive physical playground combines features and advantages of computer games and traditional playgrounds. This innovative platform will be described and experiments with children on developed Playware games will be presented in this paper.

\section{Capturing Entertainment}

There have been several psychological studies to identify what is "fun" in a game and what engages people playing computer games. Theoretical approaches include Malone's principles of intrinsic qualitative factors for engaging gameplay [5], namely 


\section{Georgios N. Yannakakis, John Hallam and Henrik Hautop Lund}

challenge (i.e. 'provide a goal whose attainment is uncertain'), curiosity (i.e. 'what will happen next in the game?') and fantasy (i.e. 'show or evoke images of physical objects or social situations not actually present') as well as the well-known concepts of the theory of flow ('flow is the mental state in which players are so involved in the game that nothing else matters') [1]. Other qualitative studies include Lazzaro's "fun" clustering — based on Malone's categorization — on four entertainment factors based on facial expressions and data obtained from game surveys on players [3]. Koster's [2] theory of fun, which is primarily inspired by Lazzaro's four factors, defines "fun" as the act of mastering the game mentally.

Following the theoretical principles reported from Malone [5], Lazzarro [3], Koster [2] and Yannakakis and Hallam [7], this paper is primarily focused on the game opponents' behavior contributions - by enabling appropriate learning patterns for the player to be further trained on [2] — to the real-time entertainment value of the game.

\section{Playware Playground}

New emerging playing technologies (e.g. computer games) have contributed to transforming the way children spend their leisure time: from outdoor or street play to play sitting in front of a screen. This sedentary style of play may have health implications [4]. A new generation of playgrounds that adopt technology met in computer games may address this issue. More specifically, intelligent interactive playgrounds with abilities of adapting the game according to each child's personal preferences provide properties that can keep children engaged in entertaining physical activity. On that basis, capturing the child's entertainment and adjusting the game in order to increase it can only have positive effects on the child's physical condition. The Playware playground adopts these primary concepts.

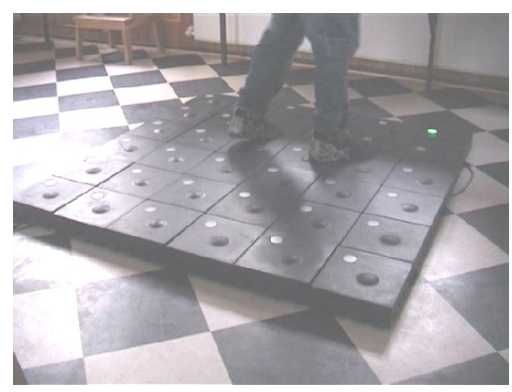

Fig. 1. A child playing the Bug-Smasher game.

\subsection{Bug-Smasher Game}

The test-bed game used for the experiments presented here is called 'Bug-Smasher'. The game is developed on a 6 x 6 square tile topology (see Fig. 1). During the game, different 'bugs' (colored lights) appear on the game surface and disappear sequentially after a short period of time. A bug's position is picked within a radius of three tiles from the previous bug and according to the predefined level of the bugs' 


\section{Capturing Entertainment through Heart Rate Dynamics in the Playware Playground}

spatial diversity. The child's goal is to smash as many bugs as possible by stepping on the lighted tiles. Different sounds and colors represent different bugs when appearing and when smashed in order to increase the fantasy entertainment factor [5].

\section{Experimental Data}

The Bug-Smasher game has been used to acquire HR physiological data and data of human judgement on entertainment. The game uses two different states ('Low' and 'High') of Malone's [5] three entertainment factors to generate a pool of 8 dissimilar games for children to play. To that end, 28 children whose age covered a range between 8 and 10 years participated in a game experiment. In this experiment, each subject plays two different games (A and B) for 90 seconds each. Each time a pair of games is finished, the child is asked whether the first game was more interesting than the second game i.e. whether $A$ or $B$ generated a more interesting game. The HR of the children is recorded in real-time by the use of a wireless ElectroCardioGram (ECG) device consisting of pulse sensors placed on the chest of the child. Out of the total number of 112 games played in this experiment, in 82 games (41 game pairs) the HR signal was properly recorded.

\section{Results}

Note that, the qualitative features of the HR signals are similar for all children that played the Bug-Smasher game: an initial rapid increase of HR during the first seconds of the game followed by a stable, but noisy, condition of high HR. Given the HR time series over 90 seconds for each game played, the following statistical parameters are computed in order to identify features of the HR dynamics that correlate with entertainment of the child while playing a game: a) The average HR, b) the variance of the HR signal, c) the maximum HR, d) the minimum HR, e) the difference between the maximum and the minimum HR f) the correlation coefficient between HR recordings and the time in which data were recorded, g) the autocorrelation of the signal, which is used to detect the level of non-randomness in the HR data and h) the approximate entropy ApEn [6] of the signal.

In addition, three different regression models were used to fit (least square fitting) the HR signal: linear, quadratic and exponential. The parameters of the regression models under investigation are: a) the slope $s$ of the linear regression $\left(h_{L}(t)=s t+\alpha\right)$ on the signal b) the parameters $\beta$ and $\gamma$ of the quadratic regression $\left(h_{Q}(t)=\beta t^{2}+\gamma t+\delta\right)$ on the signal and c) the parameters $A, B, b$ of the exponential regression $h_{E}(t)=A\left(1-e^{-b t}\right)+B$.

Results obtained show that average HR appears to be the only feature examined that is significantly correlated to entertainment $(\mathrm{c}=0.4146, \mathrm{p}=0.0057)$. The obtained effect of $E\{h\}$ appears to be commonsensical since the Bug-Smasher game belongs to the genre of action physical games where the level of engagement of the user tends to 


\section{Georgios N. Yannakakis, John Hallam and Henrik Hautop Lund}

have a significant effect on physical activity. Therefore, if we hypothesize that in such games the level of engagement correlates with average HR, it appears that the higher the average HR during a game the higher the child's perceived satisfaction and the higher his/her physical activity. Moreover, the reported [8] significant correlation between the average response time of children interacting with Playware games and entertainment is consistent with the interplay between engagement, physical activity and entertainment demonstrated here.

\section{Conclusions}

This paper introduced statistical correlations between physiological signals and children's entertainment in physical playgrounds. More specifically the average HR of the child was found to be the only statistic that correlates significantly with the child's perceived satisfaction.

The entertainment modeling approach through HR dynamics presented here demonstrates generality over the majority of action games created with Playware since additional experiments have already shown that the average HR's effect to entertainment generalizes to other dissimilar Playware designed games. Furthermore, preliminary results on a comparative study between HR signals of children playing Playware games and the HR signals of the same children exercising further strengthen the hypothesis that physical activity through entertaining games quantitatively has a dissimilar impact on HR dynamics than that of a non-entertaining form of physical activity. Given these, the proposed approach can be used for adaptation of the game's entertainment features according to the player's individual HR signal dynamics in real-time and as a baseline for validation of any intelligent interactive design applied in Playware.

\section{References}

1. Csikszentmihalyi, M.: Flow: The Psychology of Optimal Experience. New York: Harper \& Row (1990).

2. Koster, R.: A Theory of Fun for Game Design. Paraglyph Press (2005).

3. Lazzaro, N.: Why We Play Games: Four Keys to More Emotion Without Story. Technical Report, XEO Design Inc. (2004).

4. Lund, H.H., Klitbo, T., Jessen, C.: Playware technology for physically activating play. Artificial Life and Robotics Journal, no. 4, (2005) 9:165-174.

5. Malone, T.W.: What makes computer games fun? Byte (1981) 6:258-277.

6. Pincus, S.M.: Approximate entropy as a measure of system complexity. Proc. Natl. Acad. Sci. (1991) 88:2297-2301.

7. Yannakakis, G.N., Hallam, J.: A Generic Approach for Obtaining Higher Entertainment in Predator/Prey Computer Games. Journal of Game Development (2005) 1:23-50.

8. Yannakakis, G.N., Lund, H.H., Hallam, H.: Modeling Children's Entertainment in the Playware Playground. In Proceedings of the IEEE Symposium on Computational Intelligence and Games. IEEE (2006) to appear. 